\title{
An index to represent lateral variation of the confidence of experts in a 3-D geological model.
}

\author{
R.M. Lark*, S.J. Mathers, A. Marchant, A. Hulbert \\ British Geological Survey, Keyworth, Nottingham NG12 5GG \\ *Corresponding author mlark@bgs.ac.uk
}

\begin{abstract}
A Confidence Index is proposed that expresses the confidence of experts in the quality of a 3-D model as a representation of the subsurface at particular locations. The Confidence Index is based on the notion that the variation of the height of a particular geological surface represents general geological variability and local variability. The general variability comprises simple trends which allow the modeller to project surface structure at locations remote from direct observations. The local variability limits the extent to which borehole observations constrain inferences which the modeller can make concerning local fluctuations around the broad trends. The general and local geological variability of particular contacts are modelled in terms of simple trend surfaces and variogram models. These are then used to extend measures of confidence that reflect expert opinion so as to assign a confidence value to any location where a particular contact is represented in a model. The index is illustrated with an example from the East Midlands region of the United Kingdom.
\end{abstract}

\section{Introduction.}

Geologists understand the geology of a region in three dimensions, and recent technological developments allow them to represent this understanding in 3-D geological models.

Geological information in the form of 3-D models, rather than traditional 2-D maps, is now the state of the art for planning and decision making (Mathers and Kessler, 2010; Royse et al., 2010).

All geological information is subject to uncertainty, since at most sites in a region information is inferred indirectly from observations at other locations, observations which may themselves be subject to error. As a result the final model has an inevitable uncertainty. This is of interest because the model may be interpreted as indicating the subsurface positions of particular features or the volumes of particular units over a specified region. 
Engineering decisions such as the route of a tunnel or the suitability of the subsurface for particular structures should be robust given the model errors that may be expected.

Similarly, if the model is used in resource assessment or in hydrogeological modelling then the user requires some understanding of the model's uncertainyt, and how this might vary in space. For this reason the problem of how to measure and represent model uncertainty is the subject of some considerable research interest.

Where 3-D structure is predicted from observations by purely geostatistical methods a measure of uncertainty is computed directly for individual predictions (Lark and Webster 2006; Blanchin and Chilès 1993). However, most models are not generated by a statistical algorithm but rather through expert interpretation; either expert 'manual' editing of surfaces produced by mechanical interpolation, or by interpolation from cross sections interpreted by the modeller, subject to constraints (e.g. 2-D coverages for particular units) imposed by the modeller as in the GSI3D software (Kessler et al., 2009; Mathers et al., 2011).

Lark et al. (2013) report a post-hoc evaluation of uncertainty in a model produced in GSI3D by a designed experiment in which a team of modellers studied a common region. However, this approach is resource-intensive and not suitable for conditions where borehole data are sparse. It can be used to obtain benchmark statistics on model quality for particular geological terrains and settings, but is not suitable as a routine approach to quantify uncertainty in particular models.

Lelliott et al. (2009) propose a structured approach to represent the uncertainty in 3-D models. This was based on an initial analysis of factors that contribute to the uncertainty of a model in a specific geological setting produced with the GSI3D software. The factors identified include the reliability of the absolute elevations of the boreholes, the quality of the borehole logging, the drilling method, the geological complexity and the density of data. Any borehole may be given some index which reflects the quality of information it provides according to the above-listed constraints (e.g. under drilling method the quality of information was deemed best for cores obtained by sonic drilling and poorest for those obtained by cable percussion). The combination of information on the different sources of uncertainty into a single index was done by a machine-learning algorithm; this uses the various sources of information on model uncertainty as predictors of the expert score provided at a few calibration sites.

The present paper describes an index of confidence similar to that proposed by Lelliott et al. (2009). It differs from the previous measure in certain respects. It is designed specifically for models of subsurface structure inferred from subsurface observations (boreholes, seismic lines) rather than by projection from surface structure. We are concerned, therefore, 
with cases where the expert may make inferences about the height of a contact at some unobserved site by identifying a trend in the height of the same contact shown in boreholes or interpreted seismic data at other locations. We consider two general constraints on the model that the expert will form. The first is the overall geological complexity; this source of uncertainty will be small if there is strong evidence for relatively simple trends in surface heights, as may arise from a consistent dip or a dip with flexure. Lelliott et al. (2009) measure geological complexity by multiple fitting of a trend surface model to subsets of available data, which gives a measure of complexity in more general cases than ours but also risks confounding complexity as a source of uncertainty with data density since the multiple refitted surface will be more variable in areas where data are sparse, and less variable where the observations are strongly clustered in space. The second constraint on the model is local geological complexity which is, essentially, the variability of surface height about the overall trend. In our index the effect of this source of uncertainty depends on the proximity of local observations, such that the confidence in the model decays with distance from a borehole. By this simple partition of the sources of uncertainty in a model we are able to compute directly a simple Confidence Index which is interpretable in terms of a scale used to elicit information from geological experts and which varies in ways which are directly interpretable in terms of the distribution of borehole and other data and the distribution of faults. In this way the Confidence Index is entirely transparent.

In the remainder of this paper we describe the general form of our proposed Confidence Index, and the methods required to compute it. We then present a case study in which the index is computed for a model of some subsurface contacts in a part of the East Midlands of England.

\section{The Confidence Index}

\subsection{General principles}

The key idea implemented in the Confidence Index is that the confidence in the modelled surface is influenced by overall geological variability (the extent to which pronounced simple trends in the elevation of particular surfaces allow the modeller to project with some confidence beyond the range of data) and local geological complexity, which determines over what distance information in a borehole constrains the interpretation of local fluctuations around an overall trend. In order to implement the Confidence Index we use data on the elevation of the modelled surfaces of interest, either from boreholes or geophysical data. These data are then analysed to partition the observed variation in elevation of each surface into a simple trend (a polynomial of degree 1 or 2 in the 2-D coordinates) and fluctuation about the trend. The latter is treated as a Gaussian random variable which may be spatially 
correlated, that is to say the observed deviations from the trend model at two locations are more likely to be similar if the locations are close together than if they are far apart. The correlation between the deviations from the trend at any two locations declines with the distance between them to zero, which is either reached or asymptotically approached at a distance called the range of autocorrelation. The autocorrelation is modelled as a function of distance by a suitable mathematical expression. As described in more detail below the proportion of the variation in observed heights which is described by the trend component of the model characterizes the overall complexity of the modelled surface, and the dependence of the autocorrelation function for the deviations from the trend characterizes the local geological variability. The rationale for this is that if the distance to the nearest borehole from some location, $\mathbf{x}$, is longer than the range of autocorrelation of the variations of a surface about the general trend, then that borehole provides no direct information on the local geological variability at $\mathbf{x}$.

Consider four contrasting locations in a modelled area.

A. The first is a location that coincides with a logged borehole used to produce the model. It is assumed that the borehole data are of good quality, and are reliably coded. At this location there is complete confidence in the model, because it is directly supported by an observation and the Confidence Index is allocated a maximum value, $a_{1}$. One might, however, identify among all the boreholes in a region subsets which command different degrees of confidence because of factors such as age, logging quality, drilling method etc. In these circumstances one may elicit from geologists with local knowledge and experience, which includes experience of the borehole sets, values of the Confidence Index at sites that coincide with the different borehole subsets. These values may be denoted $a_{2}, a_{3} .$. all less than $a_{1}$.

B. The second location is one that coincides not with a borehole but with a point on a seismic line or some other geophysical measurement which provides information used in the model. Here the confidence in the model is enhanced by the extent to which the observation constrains the modelled surface, but it is likely that the value of the Confidence Index at such a location should be less than at a borehole location. That is because the depth of a contact at a location on a seismic line is inferred mathematically on the basis of assumptions about the seismic velocity of the units in the stack. This is an additional source of uncertainty. Once again it would be necessary to elicit from experts, with experience of geophysical measurement in the particular geological setting, a value for the Confidence Index relative to the maximum value $a_{1}$ for the best-quality borehole data. We denote this value by $b$, but 
recognize that it might be necessary to elicit more than one value for different subsets of geophysical data.

C. The third situation is a location some distance from any direct observation such that the observations only constrain the model at the location through any general trends in the height of the surfaces of interest that are identified in the data as a whole. The confidence in the model will be at a minimum at such a location, the question is what value should be ascribed to the Confidence Index in such circumstances. There are two general cases here.

C.i. In the first, the depth of the surface of interest shows no large-scale structure (dip or similar trend) across the modelled region, but is influenced by fine-scale fluctuations around a constant mean depth. Such variation is not readily predictable by the modeller.

C.ii. In the second, there is some long-range structure, such as a gentle dip across all or much of the region, which is not markedly affected by faulting. This broad-scale structure is predictable by the modeller, it represents the kinds of geological feature that can be understood and interpreted and used to make well-constrained predictions about the form of a unit at a distance from hard observations.

It is proposed that the minimum value of the Confidence Index is set to some value $c$ relative to the maximum $a_{1}$. Different values of $c$ may be allocated according to the strength of evidence for a simple trend in the data for a particular surface. This is described in detail in section 2.3 below.

D. Here we consider a location near one or more boreholes or geophysical observations. The datum provides some information about local geological complexity, which improves the confidence in the model relative to value $c$ but confidence will be less than at the location of the observation itself. In this study we use the autocorrelation function for deviations from the trend surface to determine the rate of decay of the Confidence Index from a local maximum ( $a$ or $b$ ) to the minimum value $(c)$ which it reaches at a distance equivalent to the range of the autocorrelation. That is to say, we assume that the influence of a borehole on our confidence in the model at some neighbouring location is proportional to the correlation that we expect between the variations of surface height about the overall trend between the borehole and the location of interest. Note that we assume that no fault intervenes between the borehole and the location under consideration, so it is the correlation with the nearest borehole such that the straight line to the borehole intersects no faults which determine the value of the Confidence Index at any location.

\subsection{Definition and implementation}


The first step to implement this index is statistical analysis of available data on the heights of target surfaces to assess their general and the local geological complexity. The second is an elicitation from experts, familiar with the geological setting and with any geophysical data used, of appropriate values for the parameters of the index, appropriate values of $\left\{a_{1}, a_{2} .\right.$. ; $\left.b_{1}, b_{2 . . ;} c_{1}, c_{2} . . ;\right\}$ as described in the previous section.

2.2.1 Statistical analysis: exploratory data analysis The first step in the data analysis is the exploratory analysis of the data to investigate their variability. The data are on heights of target surfaces, and may be obtained from boreholes, from interpretation of observations on seismic lines or both. Geophysical data may be particularly appropriate for this analysis because they are likely to be numerous. The data are examined for evidence of spatial trends. To do this one may plot surface height against eastings and northings separately, and supplement these plots with classified post-plots in which the locations of observations are displayed on a 2-D map with colour or a symbol indicating a range of values to which each observation belongs.

Another exploratory tool to examine evidence for a trend is to compute the empirical variogram of the data. This is estimated by

$$
\hat{\gamma}_{\mathrm{M}}(\mathbf{h})=\frac{1}{2 N(\mathbf{h})} \sum_{i=1}^{N(\mathbf{h})}\left\{z\left(\mathbf{x}_{i}\right)-z\left(\mathbf{x}_{i}+\mathbf{h}\right)\right\}^{2}
$$

where $N(\mathbf{h})$ pairs of observations among the available data are separated by an interval $\mathbf{h}$, called the lag, and $z(\mathbf{x})$ denotes an observation at location $\mathbf{x}$ (Matheron, 1962). Note that $\mathbf{h}$, the lag, is a vector with a distance and a direction. The variogram can be computed for random variables on some simple assumptions (Webster and Oliver, 2007). In this particular setting we are not interested in variation in surface height due to faulting and so, throughout the analyses described here, the variogram is estimated only from pairs of observations such that the straight line joining those observations intersects no faults.

The variogram is displayed by plotting the values, determined from Equation (1), against the lag distance. Key features of a typical empirical variogram are illustrated in Figure 1. The variogram generally increases with lag distance, commonly to some upper bounding value, called the sill variance, which it reaches, or approaches, at a lag distance called the range. Observations separated by a distance longer than the range can be regarded as statistically independent. If the variogram of a variable reaches a sill variance or approaches it asymptotically then this tells us that it is possible to write an autocorrelation function for that variable, as we explain in detail in section 2.2.3 below. 
An empirical variogram often appears to have a non-zero intercept at lag zero. This is called the nugget variance, and represents the variation of the variable which occurs at very fine scales not resolved by the spatial sampling.

If the variogram depends only on the lag distance it is said to be isotropic. An anisotropic variogram depends only on the lag direction. There are two kinds of anisotropy. Geometric anisotropy can be modelled by a simple transformation of the coordinate system; and variograms different directions have a common sill. If the variograms for different directions have different sills, this is called zonal anisotropy and it is not so readily-modelled (Webster and Oliver, 2007).

If data are affected by a long-range trend, then the variogram will tend to increase parabolically with lag distance.

2.2.2 Statistical analysis: statistical modelling of a trend We consider a polynomial trend model up to a full quadratic:

$$
z=\beta_{0}+\beta_{1} x+\beta_{2} y+\beta_{3} x^{2}+\beta_{4} y^{2}+\beta_{5} x y,
$$

where $z$ is the depth of the surface of interest and $x$ and $y$ denote eastings and northings respectively. This quadratic model could accommodate a simple trend such as a dip or a dip with flexure. We do not necessarily include all terms in this model. We propose that the final trend model is selected by searching through all subsets of the full model in Equation (2) (with 5 predictors) to find the best-fitting subset according to the Akaike information criterion (AIC), Akaike (1973). The general method is described by Miller (1990), we used the implementation in the RSEARCH procedure in Genstat (Payne, 2010) to do this.

Having fitted a trend model we may evaluate the overall strength of evidence for a trend. If the exploratory analysis and the model-fitting indicate that there is no trend, then the parameter $c$ for the Confidence Index takes a minimum value $c_{1}$. We propose that values of $c$ are elicited from experts according to the following criteria:

$c_{1} \quad$ The smallest possible value, for variables which show no spatial trend.

$c_{2} \quad$ The background value for variables which show weak spatial trend.

$c_{3} \quad$ The background value for variables which show moderate spatial trend.

$c_{4} \quad$ The background value for variables which show strong spatial trend.

The strength of the trend may be judged from the coefficient of determination of the fitted trend model, $\mathrm{R}^{2}$. This is a measure of the proportion of variation in variable $z$ which is accounted for by the trend model. The following rule of thumb is suggested: 
Weak trend $\quad R^{2} \leq 0.25$

Moderate trend $\quad 0.25<\mathrm{R}^{2} \leq 0.75$

Strong trend $\quad R^{2}>0.75$

\subsubsection{Statistical analysis: computing the autocorrelation function for local geological} variability Having selected a trend model, the variability about the trend may be examined by computing the empirical variogram for discrete lags, using Equation (1), for the residuals from the trend, i.e. the difference at each observed location between the trend model and the observed height of the target surface. A standard second-order stationary variogram function was fitted to the estimates by weighted least squares as described by Webster and Oliver (2007) and implemented in the MVARIOGRAM procedure in Genstat (Payne, 2010). The model variogram takes the general form:

$$
\begin{array}{rlrl}
\gamma(h) & =g_{0}+g_{1} s(h) & & h>0 \\
& =0 & h & =0,
\end{array}
$$

where $h$ is a lag distance and $s(h)$ is a function of distance. The two terms $g_{0}$ and $g_{1}$ are variances. The term $g_{0}$ is the nugget variance, introduced in section 2.2.1 above. The sum $g_{0}+g_{1}$ is the sill variance, the upper bounding value of the variogram. In this study we found the spherical and circular variogram models to be appropriate, these have a single parameter, the range at which the autocorrelation goes to zero (Webster and Oliver, 2007). Once a second-order stationary variogram model has been fitted one may compute the autocorrelation of observations separated by lag distance $h$ as

$$
\rho(h)=1-\left\{\gamma(h) /\left(g_{0}+g_{1}\right)\right\}
$$

Note that the autocorrelation at distance zero is 1 , and that it goes to zero at the range of autocorrelation.

The form of the autocorrelation function is informative for our purpose in defining a confidence index. Consider a case in which the only variation in the depth of the surface of interest is represented by the trend model (Equation 2) and independent random noise. In this case the term $g_{1}$ in Equation (3) above is zero, all the variation about the trend is in the nugget term $g_{0}$. The variogram is therefore a constant for $h>0$, which means that the autocorrelation function is 1 at $h=0$, and 0 for $h>0$. Let us consider the significance of entirely independent variation about the trend. What this tells us is that the departure of the height of the surface of interest at a borehole is entirely uncorrelated with variation about the trend at any other location, and so our confidence in the model at a location a short distance 
from the nearest borehole is no greater than at another location a longer distance from the nearest borehole. Our confidence declines from $a$ to $c$ as soon as we move some distance $h>0$ from a borehole, just as $\rho(h)$ declines from 1 to 0 .

More usually we would expect to see some spatial correlation in the variation of the elevation of a surface about a general trend (e.g. Lark and Webster, 2006). That means that $g_{1}>0$, and so the autocorrelation declines to zero at some distance, the range of the autocorrelation function. In this case the knowledge that the surface of interest is somewhat higher (for example) than the trend surface at a particular borehole is informative about the likely elevation at locations which are closer to the borehole than the range. The closer a location of interest is to the nearest borehole the larger the value of the autocorrelation for the lag distance between them, reflecting the extent to which the borehole is informative about conditions at that location, and so provides us with confidence in the model.

\subsection{Elicitation of parameters of the Confidence Index.}

The discussion of the Confidence Index above shows that we require parameters which reflect the geologist's expert judgement on uncertainty. These may be obtained by the following questionnaire:

i. Do you regard all borehole observations used in the production of this model (whether directly in cross-sections or to support interpretations) as being of uniform quality? If not, then indicate how you would divide the boreholes into subsets which are all internally of fairly uniform quality.

ii. If there are $n_{\mathrm{b}}$ such subsets of boreholes, then indicate how you would rate the reliability of boreholes in each subset, if the best possible boreholes were given rating 10 , and boreholes with no information content at all were rated 0 .

This will provide us with a set of values $a_{1}, a_{2}, . . a_{n b}$.

iii. Do you regard all geophysical observations used in the production of this model (whether directly in cross-sections or to support interpretations) as being of uniform quality? If not, then indicate how you would divide the available observations into subsets which are all internally of fairly uniform quality.

iv. If there are $n_{\mathrm{g}}$ such subsets of geophysical data, then indicate how you would rate the reliability of data in each subset, if the best possible borehole observation were given rating 10 , and data with no information content at all were rated 0. 
This will provide us with a set of values $b_{1}, b_{2}, . . b_{\mathrm{ng}}$.

v. Consider a location in the modelled area remote from any borehole or geophysical data where the model is dependent entirely on interpretation of any trend in the surfaces of interest. How would you rate confidence in the model at such a location in each of the following circumstances, relative to confidence at the location of a borehole of best possible quality which has score 10 ?

1. When there is no spatial trend to project from available observations, so the only information these provide is on the mean height of the surface.

2. When there is a weak spatial trend to project from available observations.

3. When there is a moderate spatial trend to project from available observations.

4. When there is a strong spatial trend to project from available observations.

This will provide us with a set of values $c_{1}, c_{2}, c_{3}, c_{4}$.

This entire questionnaire may be sent to all available experts, or questions $\mathrm{i}$, ii, and $\mathrm{v}$ might be considered by geologists with modelling experience whereas iii and iv are considered by geophysicists with experience of the particular geophysical methods in comparable geological settings.

The information above allows the Confidence Index to be computed for a given surface at any location. For simplicity we consider the case where the autocorrelation function is isotropic so its argument is a scalar (distance). Consider some location $\mathbf{x}$, let the distance to the nearest borehole, not separated from $\mathbf{x}$ by a fault, be $d^{\prime}{ }_{b}$ and let this borehole belong to quality subset $i$ of the available boreholes, as obtained from question (ii) of the elicitation. Let the distance to the nearest geophysical observation, not separated from $\mathbf{x}$ by a fault, be $d^{\prime}{ }_{g}$ and let this borehole belong to quality subset $j$ of the available data, as obtained from question (iv) of the elicitation. Let $c$ be the minimum value of the Confidence Index, obtained by defining the strength of the trend from the exploratory analysis and the experts' answers to question ( $v$ ) of the elicitation. The value of the Confidence Index is then given by

$$
I(\mathbf{x})=\max \left\{c+\left(a_{i}-c\right) \rho\left(d_{\mathrm{b}}^{\prime}\right), c+\left(b_{j}-c\right) \rho\left(d^{\prime}{ }_{\mathrm{s}}\right)\right\}
$$

It can be seen from this expression that the local maximum values of $a_{i}$ or $b_{j}$ will be returned at the locations of boreholes or seismic observations respectively, and the background value of $c$ at any location where the distance to the nearest observation exceeds the range of 
autocorrelation. At other locations the Confidence Index will take intermediate values, depending on the proximity of observations.

\section{Case study}

\subsection{The study area and model.}

In order to illustrate the use of the Confidence Index data have been used from the East Midlands region of the UK specifically a portion of the BGS 1:250,000 resolution bedrock geology model of the region. The entire East Midlands model covers approximately 15000 $\mathrm{km}^{2}$ stretching from Nottinghamshire and Leicestershire in the west to the Lincolnshire coast, the Wash and the western parts of North Norfolk (Pharaoh et al., 2011). The area selected for this study has British National Grid co-ordinates as follows: lower left (south west corner) 460290 and top right (north east corner) 540340.

The geology of the study area includes three main geological components or structural levels: Mesozoic cover sediments (Triassic, Jurassic and Cretaceous), Carboniferous and Basement. From the surface there is a shallow, eastward-dipping, sparsely-faulted Mesozoic sedimentary cover sequence, several hundred metres in thickness and predominantly of Jurassic and Triassic age. These deposits rest on the Variscan Unconformity and overlie more intensely faulted and folded Carboniferous strata containing hydrocarbon resources and source rocks. Locally these deposits exceed a kilometre in thickness and rest on the Caledonian Unconformity. Below this unconformity, a third level composed of tightly folded and faulted metasedimentary and metavolcanic Lower Palaeozoic and Neoproterozoic rocks are present, cut by several large granitic intrusions.

The stratigraphy of the East Midlands region, with the surfaces produced in the construction of the model shown in red, is shown in Figure 2. These surfaces comprise the major stratigraphic boundaries, marker horizons and unconformities that are consistently identifiable in subsurface data in the region. In this paper we focus on two surfaces in the model: the Variscan Unconformity and the top of the Dinantian Carboniferous limestones (a unit shown in Figure 2 which Pharaoh et al (2011) divided into the Tournaisian and Viséan).

The East Midlands model was built using the GOCAD ${ }^{\circ}$ software (http://www.gocad.org/w4/) following initial processing and interpretation of the seismic reflection data, and earlier modelling and hand contouring of stratigraphic surfaces. The model is supported and constrained by these interpreted data described in Pharaoh et al. (2011). The distribution of boreholes contributing to the model within the study area is shown in Figure 3 , there is a 
strong concentration of boreholes in the north-western quadrant reflecting exploration for hydrocarbon resources within the deep Carboniferous basin.

\subsection{Geophysical data}

The locations of 2-D seismic reflection lines used in modelling within the study area are shown in Figure 3. As with the boreholes, there is a strong concentration of data in the north-western quadrant of the area. The data contains regional hydrocarbon exploration data, combined with Coal Authority high-resolution seismic data of more localised extent. The seismic data for the model were sourced from the DECC (http://og.decc.gov.uk/en/olgs/cms/data maps/data release/data release.aspx) and the United Kingdom Onshore Geophysical Library (UKOGL) (http://www.ukogl.org.uk/seismiccoverage.htm)websites. Horizons mapped from the seismic data in two-way-travel-time (TWTT) were converted to depth using velocity functions based on depths and times in reference boreholes that have velocity data available.

\subsection{Geostatistical analysis}

Seismic data are the most widespread and numerous, so these were used in the statistical analysis to evaluate evidence for simple trends in elevation of the target surfaces and for geostatistical analysis of the local variability about the trend. This was undertaken on seismic data for the Variscan Unconformity (UVAR) surface and the top of the Dinantian strata (DIN). Figure 4 shows post-plots of the seismic data. A post plot shows the spatial distribution of data with symbols that indicate recorded values. Here the colour coding of the symbols indicates quintiles of the data, that is to say the range of values is divided into 5 intervals, each of which contains $20 \%$ of the observations. The positions of faults cutting these surfaces are also shown on the post plots.

The Variscan Unconformity, shows a pronounced trend with the surface becoming deeper to the north-east. This is apparent in the post plot (Figure 4a) The top of the Dinantian, shows a more complex structure, it is deepest in the north-western part of the study area.

When trend surface models were fitted to the data for each surface, a full quadratic model was selected in each case on the basis of the AIC,that is a model with all the terms in Equation (2). Table 1 presents the coefficient of determination of the fitted trend model $\left(R^{2}\right)$ values for the trend model for both surfaces and the inference drawn from that about the strength of the trend and so the value of the term $c$ in the Confidence Index. Figure 5 shows the empirical variograms for the standardized residuals from both surfaces. Note that both show some degree of directional dependence, although this is small for the top Dinantian 
surface (DIN), and is small at lag distances of up to $2 \mathrm{~km}$. In both cases the directional dependence is seen in the sill variance, so the anisotropy is zonal. For this reason isotropic variograms were estimated for use in the Confidence Index.

The authorized variogram models were fitted to the isotropic estimates, and these are shown in Figure 6. The parameters of the variograms are shown in Table 1. Recall from section 2.2.3 that the distance parameter $r$ is the range of the circular and spherical variograms, the distance at which the autocorrelation goes to zero.

\subsection{Elicitation of parameters of the Confidence Index}

The elicitation procedure described in section 2.3 above was followed in two steps. In the first step we obtained values of parameters $a$ and $c$ from a geologist with substantial modelling experience and familiarity with the target units. This expert provided values for the parameters $a$ and $c$ which are presented in Table 2. Parameter $b$ was elicited from a group comprising a mix of the three geological modellers and three geophysicists who have worked extensively with seismic interpretation who were asked to provide a confidence rating for the available seismic data, relative to parameter a with a score of 10 . This was set following almost unanimous agreement to 7 for all locations and surfaces on the available seismic lines.

\subsection{Computation of the Confidence Index.}

The procedure to determine the value of the Confidence Index for a given surface at location $\mathbf{x}$ consists of the following steps.

1 Given the target surface, identify the variogram model and the values of its parameters from Table 1. Given the strength of evidence for a trend, identify the appropriate value of parameter $c$.

2. Measure $d_{\mathrm{b}}$, the distance from $\mathbf{x}$ to the nearest borehole which proves the surface of interest such that the line from $x$ to the borehole does not cross a fault which cuts the surface.

3. Measure $d_{\mathrm{s}}$, the distance from $\mathbf{x}$ to the nearest seismic datum which reports a depth for the surface of interest such that the line from $\mathbf{x}$ to the datum does not cross a fault which cuts the surface.

4. Using the variogram model type and parameters extracted in step 1, and Equation (4) 
above compute the autocorrelation over distance $d_{\mathrm{b}}, \rho\left(d_{\mathrm{b}}\right)$.

5. Using the variogram model and parameters extracted in step 1, and Equation (4) above compute the autocorrelation over distance $d_{\mathrm{s}}, \rho\left(d_{\mathrm{s}}\right)$.

6. Using the elicited values of $a$ and $b$, the value of $c$ identified at step 1 and the autocorrelations computed at steps (4) and (5), compute the value of the Confidence Index using Equation (5) above

Calculation of the Confidence Index was implemented using ESRI's ArcGIS 10.0. This required the production of some newly-written code which was validated by comparing its output with manually-calculated values.

The resulting elevation grids and corresponding Confidence Index plots are shown for the Variscan Unconformity (Figure 7) and the top of the Dinantian strata (Figure 8).

The elevation grid for the Variscan Unconfomity shows that the surface is present across the entire study area. The overall pattern shows a fall from the southwest towards the northeast of the area (Figure 7a), in detail the pattern is disrupted by some of the major W-E trending faults. The corresponding Confidence Index plot (Figure 7b) shows small values in the south and east with local large values induced by individual deep boreholes or clusters. In the heavily-faulted Carboniferous basin in the northwest of the area Confidence Index is generally large because of the density of seismic profiles and deep boreholes; the truncation of the confidence index values at faults is also well-illustrated.

The Dinantian strata are restricted to the Carboniferous Basin in the northwest of the study area. The elevation grid of the unit top exhibits strong fault control on the top Dinantian surface which descends to depths of about $1 \mathrm{~km}$ below Ordnance Datum in the extreme northwest (Figure 8a). The corresponding Confidence Index plot (Figure 8b) shows large values in red associated with the deep borehole data in that area and the alignment and density of seismic lines (shown by linear runs and coalescing area of green values respectively. As discussed above the high confidence values associated with the boreholes are truncated by the faults. 


\section{Discussion}

The Confidence Index that we propose in this paper is a means to summarize and present the sources of uncertainty in surfaces modelled on the basis of subsurface information from boreholes and geophysics. It is based on the concept that the modelling process entails the identification of broad geological variation, which can be identified with simple trends in elevation which may be projected to some distance form observations; and local geological variation, represented by variations in elevation about such simple trends. Although statistical analysis of data is used to express the uncertainty in models arising from local geological variation and broad trends, the Confidence Index is not a statistical measure of uncertainty, it is appropriate for geological models based on expert interpretation of subsurface data.

The purpose of this confidence index is to display spatially the implications for model uncertainty of particular expert judgements on the reliability of data sources, and statistical analysis of the strength of simple trends in geological surfaces and the autocorrelation of variations about these trends. The index is transparent in that its minimum and maximum values are explicitly based on expert opinion, which can be challenged and discussed, and the lateral variations of the index depend on the distribution of boreholes and the spatial statistical model fitted to available data in accordance with established and robust methodology.

The purpose of this paper was to illustrate the potential of this index. One aspect of the procedure which could be developed is the elicitation of parameters from experts. Here we used values which were agreed by the whole expert set, or represented a majority opinion. Variability of expert opinion could be taken into account by alternative elicitation methods. One approach, which might be particularly appropriate for the elicitation of an index, which is more prone to subjective differences of interpretation between experts than a variable such as a measureable rock property, is to define the index parameters as fuzzy numbers and to use methods of elicitation based on fuzzy logic (Zadeh, 1965) to obtain them. An interesting recent application of these ideas in environmental risk management is provided by Page et al. (2012).

The study of uncertainty in 3-D geological modelling is at an early stage, and this Confidence Index should be developed in the future. In particular we need a more sophisticated understanding of how different steps in the modelling work flow introduce uncertainty into the final model, and how the error at one stage is propagated by subsequent operations. Without this understanding it may be difficult to relate the actual uncertainty in the model at 
some location to evident sources of such uncertainty such as proximity to boreholes or to outcrop (Lark et al., 2013). We also note that this Confidence Index is explicitly designed for models based on subsurface information, boreholes and geophysical data. In other geological settings such information may be very sparse and modelling may depend heavily on the projection to depth of observed structures at the surface. A different approach is required to represent uncertainty in such circumstances.

\section{Conclusions}

In this paper we have introduced a confidence index which can be evaluated across a modelled region in a transparent and repeatable way to indicate the degree of confidence we can have in a model given some expert-elicited values and a simple concept of model uncertainty which depends on broad geological structure and local variability. The index has been implemented for a geological model typical of geological conditions in many parts of the UK, based on an extract from the BGS 1:250,000 resolution bedrock model of the East Midlands.

\section{Acknowledgements}

This paper is published with the permission of the Director of the British Geological Survey (NERC). 


\section{References}

Akaike, H., 1973. Information theory and an extension of the maximum likelihood principle. In: Petrov, B.N. \& Csaki, F. (Eds.), Second International Symposium on Information Theory. Akademiai Kiado, Budapest, pp. 267-281.

Blanchin, R., Chilès, J.P. 1993. The Channel Tunnel: geostatistical prediction of the geological conditions and its validation by the reality. Mathematical Geology 25, 963-974.

Kessler, H., Mathers, S.J., Sobisch, H.-G. 2009. The capture and dissemination of integrated 3D geospatial knowledge at the British Geological Survey using GSI3D software and methodology. Computers \& Geosciences 35, 1311-1321.

Lark R.M., Mathers, S.J., Thorpe S., Arkley, S.L.B., Morgan D. J., Lawrence, D.J.D. 2013. A statistical assessment of the uncertainty in a 3-D geological framework model. Proceedings of the Geologists' Association. 124, 946-958.

Lark, R.M., Webster, R. 2006. Geostatistical mapping of geomorphic variables in the presence of trend. Earth Surface Processes and Landforms 31, 862-874.

Lelliott, M.R., Cave, M.R., Wealthall, G.P. 2009. A structured approach to the measurement of uncertainty in 3D geological models. Quarterly Journal of Engineering Geology and Hydrogeology. 42, 95-105.

Matheron, G. 1962. Traité de Géostatistique Appliqué, Tome 1. Memoires du Bureau de Recherches Géologiques et Minières, Paris.

Mathers, S.J., Kessler, H. 2010. Shallow sub-surface 3D geological models for Earth \& Environmental Science decision making. Environmental Earth Science 60, 445-448.

Mathers, S.J., Wood, B., Kessler, H. 2011. GSI3D 2011 software manual and methodology. British Geological Survey Internal Report. OR/11/020. 152 pp.

Miller, A.J. 1990. Subset selection in regression. Chapman and Hall, N.Y.

Page, T., Heathwaite, A.L., Thompson, L.J., Pope, L., Willows, R. 2012. Eliciting fuzzy distributions from experts for ranking conceptual risk model components. Environmental Modelling and Software. 36, 19-34.

Payne, R.W. (Ed.), 2010. GenStat Release 13 Reference Manual Part 3: Procedure Library PL21. VSN International, Hemel Hempstead, Hertfordshire. 
Pharaoh, T.C., Vincent, C.J., Bentham, M.S., Hulbert, A.G., Waters, C.N., Smith N.J.P. 2011 Structure and evolution of the East Midlands region of the Pennine Basin. British Geological Survey Subsurface Memoir 144pp.

Royse, K.R., Kessler, H., Robins, N.S., Hughes, A.G., Mathers, S.J. 2010. The use of 3D geological models in the development of the conceptual groundwater model. Zeitschrift der Deutschen Gesellschaft für Geowissenschaften. 161, 237-249.

Webster, R. \& Oliver, M.A., 2007. Geostatistics for Environmental Scientists, 2nd Edition. John Wiley \& Sons, Chichester.

Zadeh, L. 1965. Fuzzy sets. Information and Control. 8, 338-353. 


\section{Figure Captions}

1. A hypothetical empirical variogram with key features indicated.

2. The generalised stratigraphy of the study area with the surfaces modelled shown in red.

3. Distribution of boreholes and 2-D reflection seismic lines within the study area used in the construction of the East Midlands model. The observations shown here are restricted to those that prove the modelled surfaces. Coordinates in this map and subsequent ones are in metres relative to the origin of the British National Grid.

4. Post plots of seismic data and faults for each surface. Coordinates are in metres relative to the origin of the British National Grid and Ordnance Datum (a) The Variscan Unconfomity and b) the top of the Dinantian. The colour code indicates the depth of the surface relative to Ordnance Datum (scale is for quintiles of the distribution). The black symbols indicate faults.

5. Empirical variograms of residuals from fitted trend models for seismic data on the depth of the Variscan Unconformity (UVAR) and the top of the Dinantian (DIN) surfaces. The variograms are obtained only from comparisons between pairs of points that do not cross faults. An empirical variogram plot is presented as a plot of a variance against lag distance (in metres). Different symbols indicate the lag directions.

6. Isotropic empirical variograms of residuals from a quadratic trend surface with fitted parametric models for the Variscan Unconformity (UVAR) and the top of the Dinantian (DIN) surfaces

7. The elevation grid for (a) the Variscan Unconformity surface and (b) the corresponding Confidence Index plot. White space indicates that the surface is not present in the model. Where the surface is faulted it may be shown as absent from individual raster cells where the fault is at the centre of the cell.

8. The elevation grid for (a) the top of the Dinantian (DIN) surface and (b) the corresponding Confidence Index plot. White space indicates that the surface is not present in the model. Where the surface is faulted it may be shown as absent from individual raster cells where the fault is at the centre of the cell. 


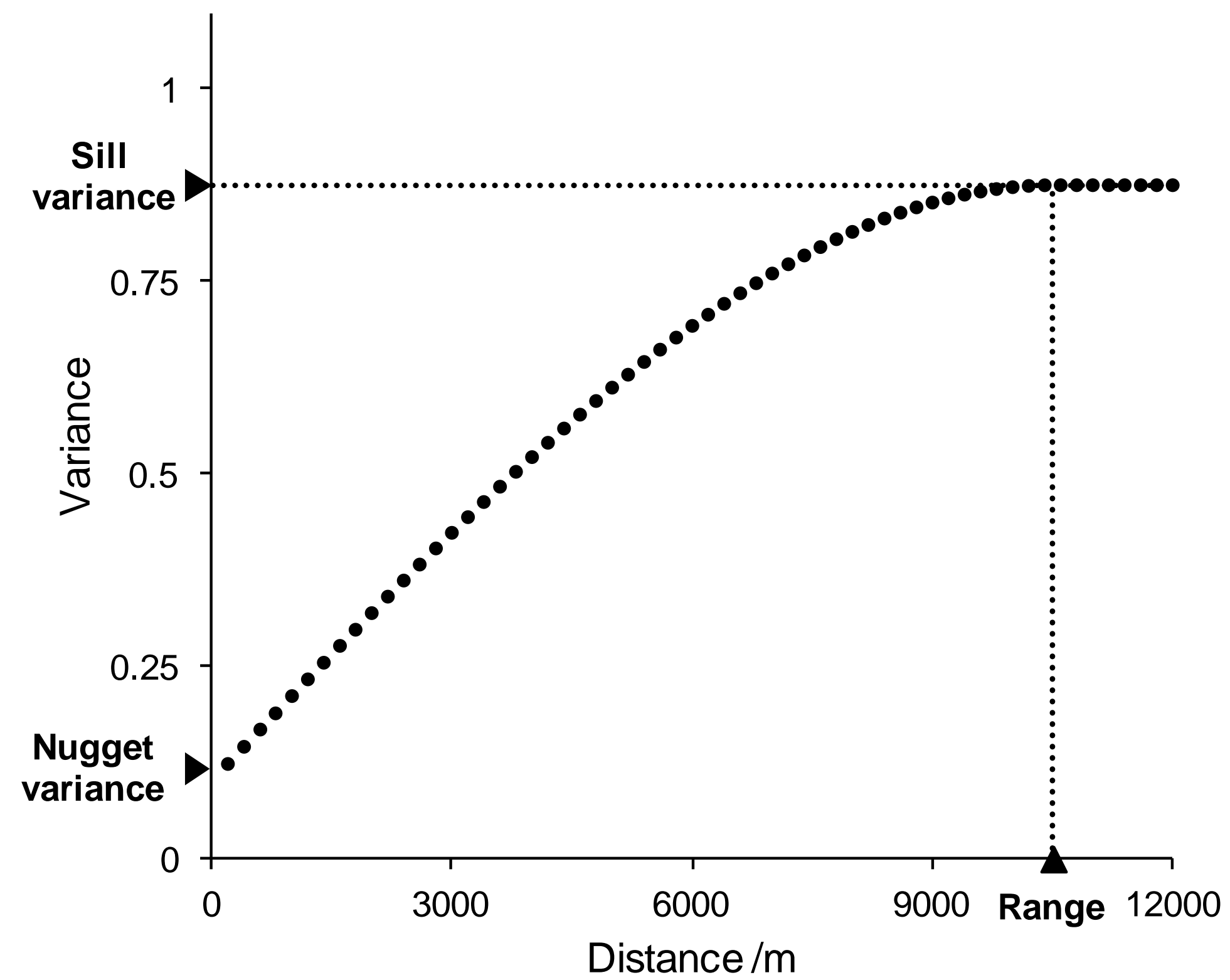


UPPER JURASSIC

MIDDLE JURASSIC

MESOZOIC COVER SEQUENCE
CARBONIFEROUS

BASIN

SEQUENCE

BASEMENT 


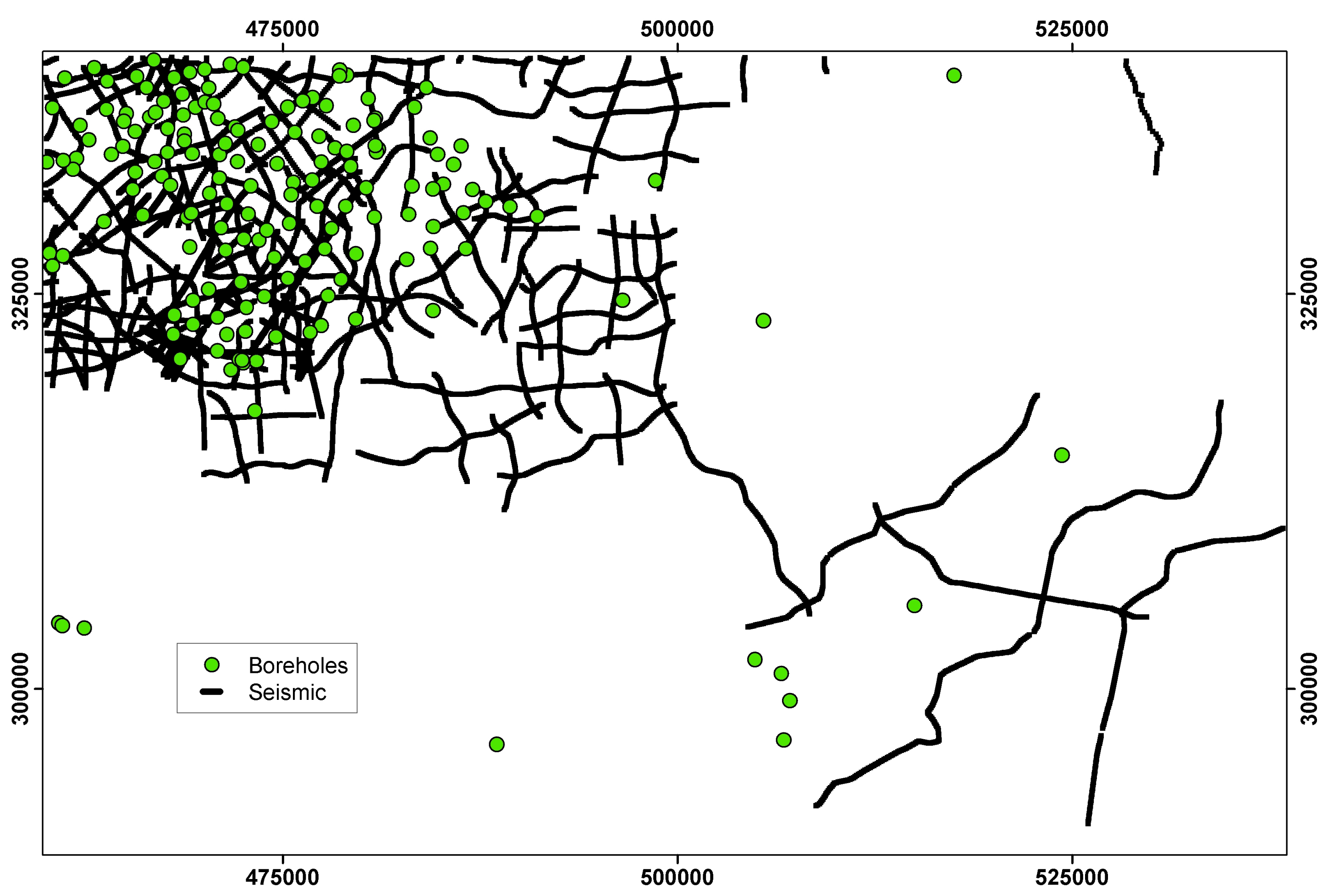


(a)

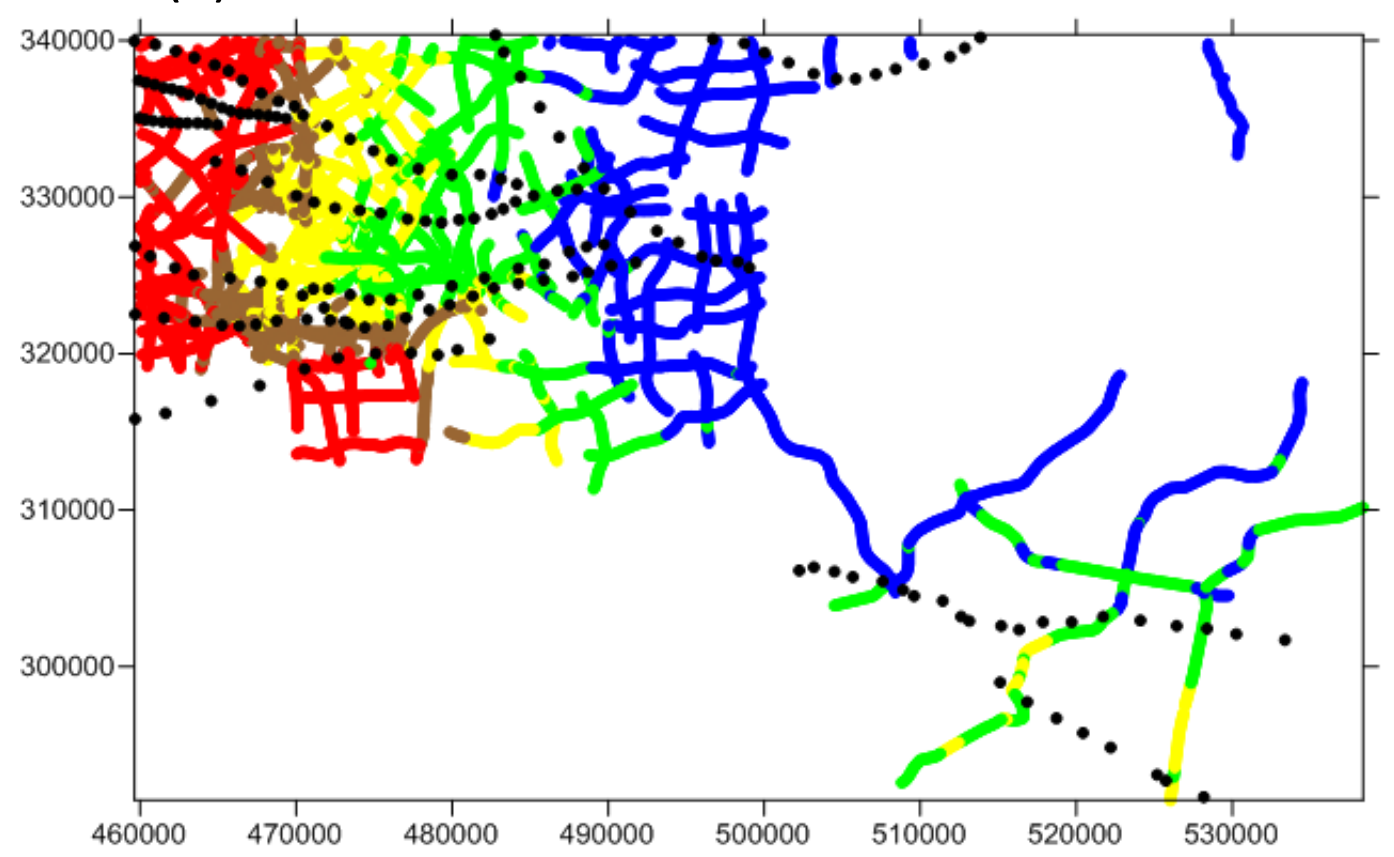

-930.7 to -479.8
-479.8 to -363.7
-363.7 to -301
-301 to -250.1
-250.1 to -70.69

(b)

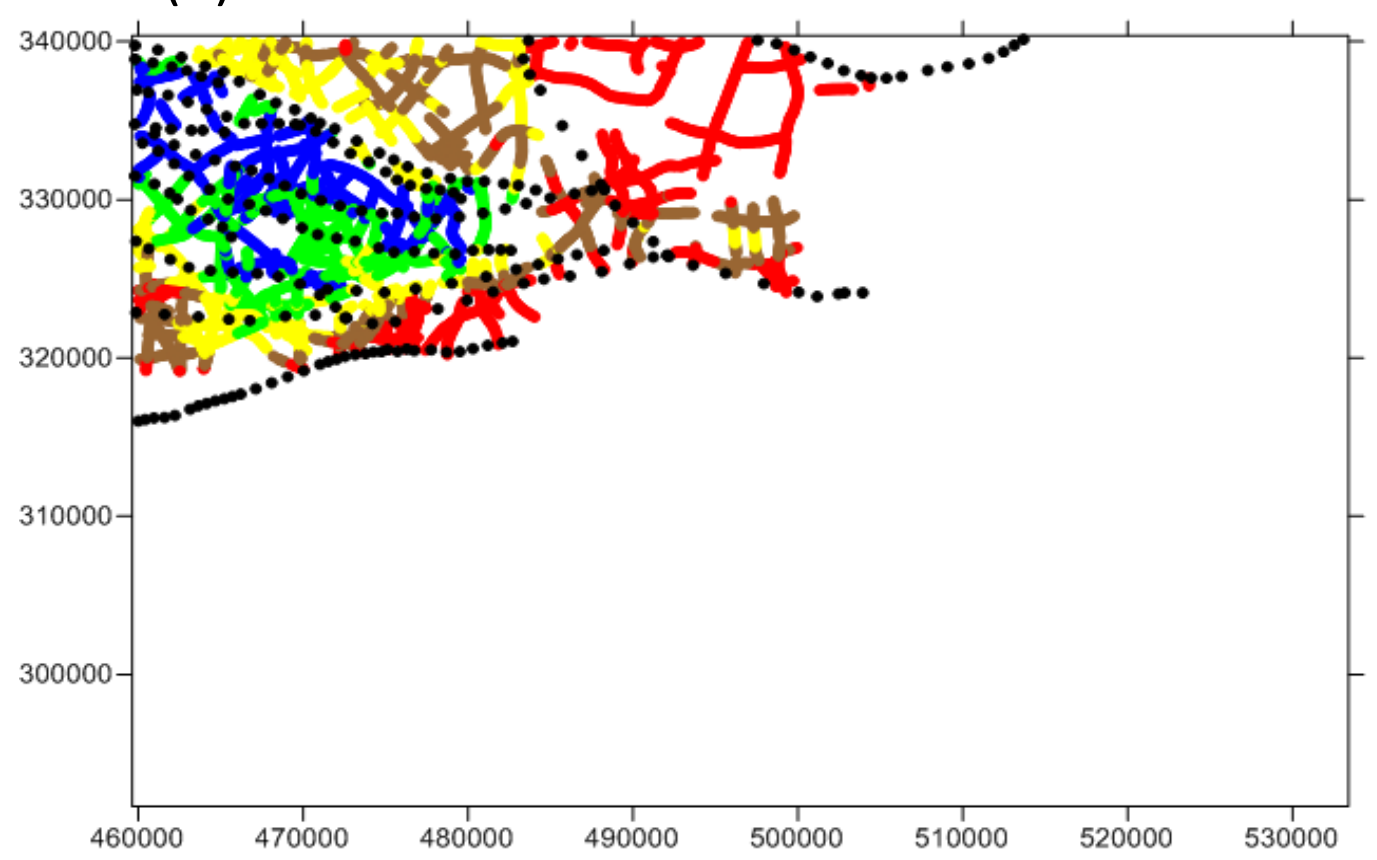

Depth /m AOD

-1634.6 to -1273.9

-1273.9 to -1133.2

-1133.2 to -940.3

-940.3 to -803.4

-803.4 to -402.7 


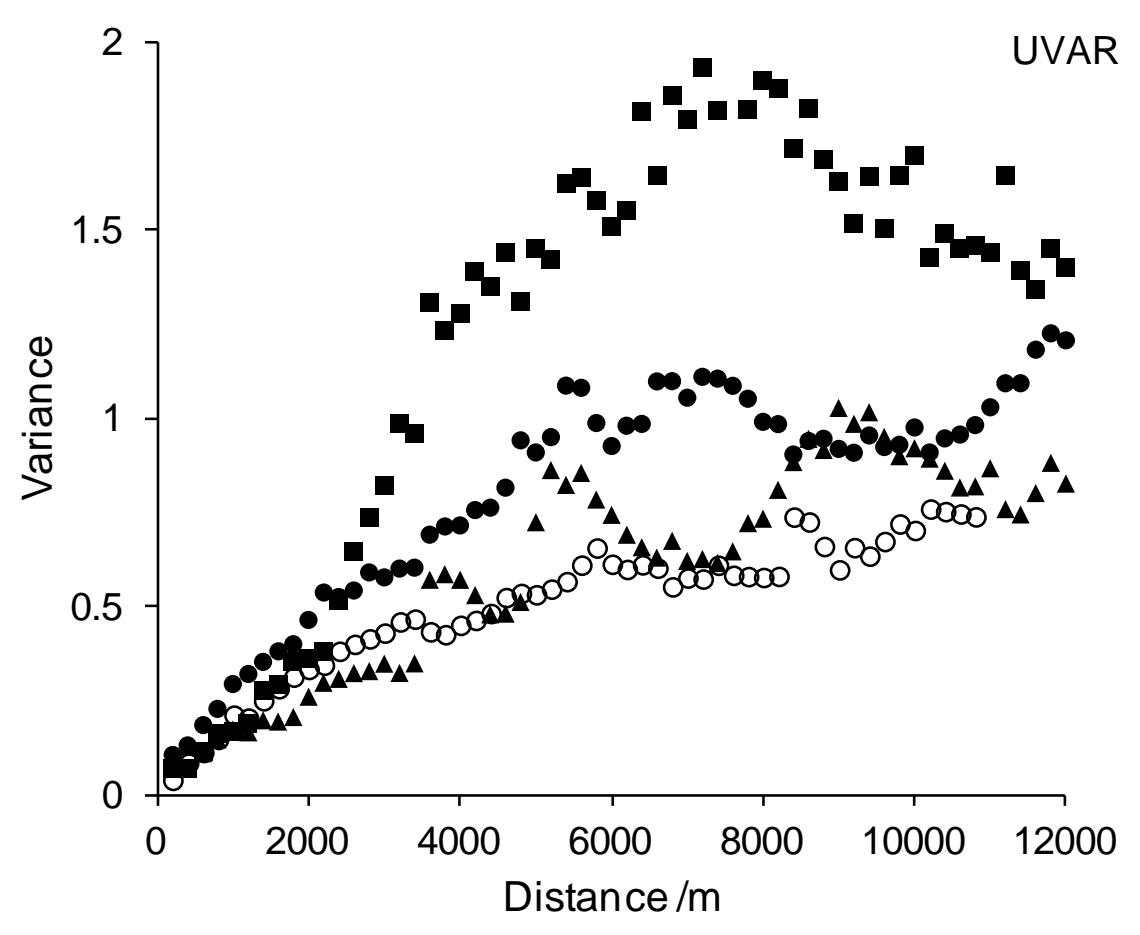

- N-S

O NE-SW

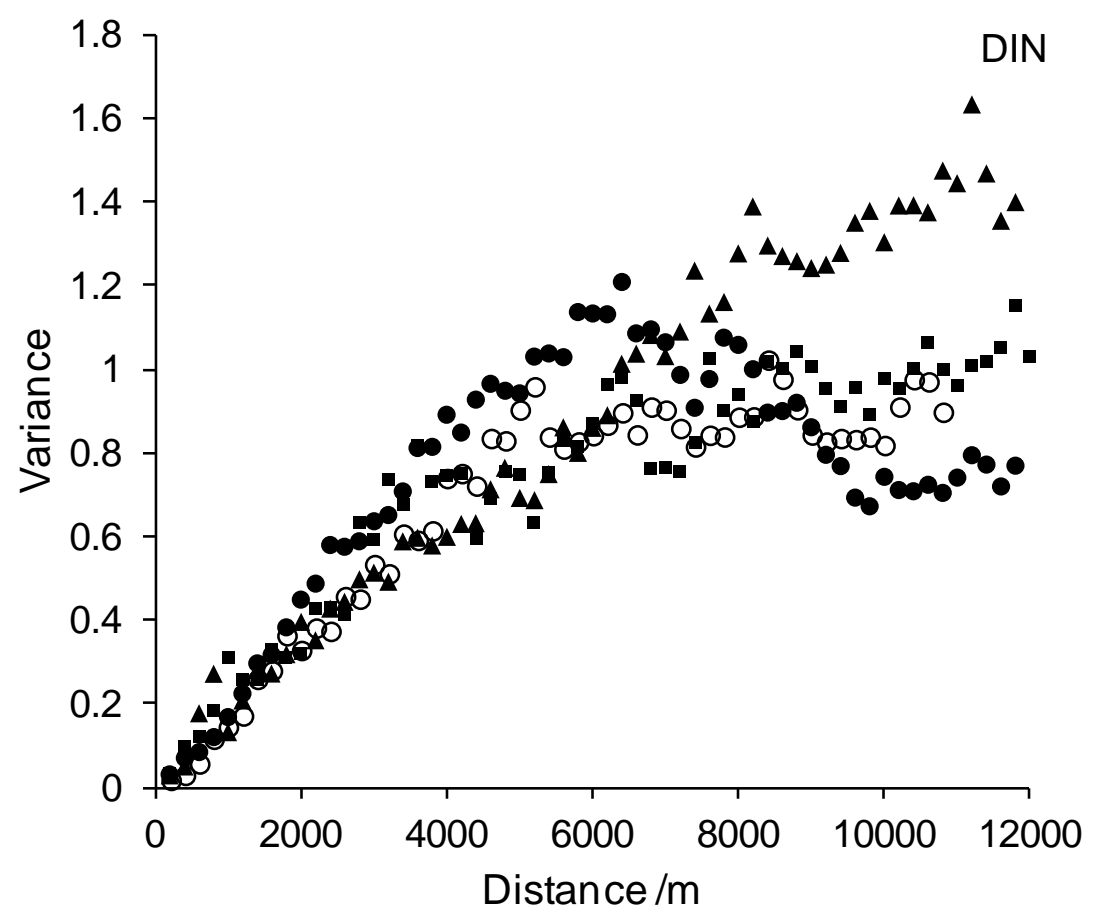

$\Delta \mathrm{E}-\mathrm{W}$

- SE-NW 

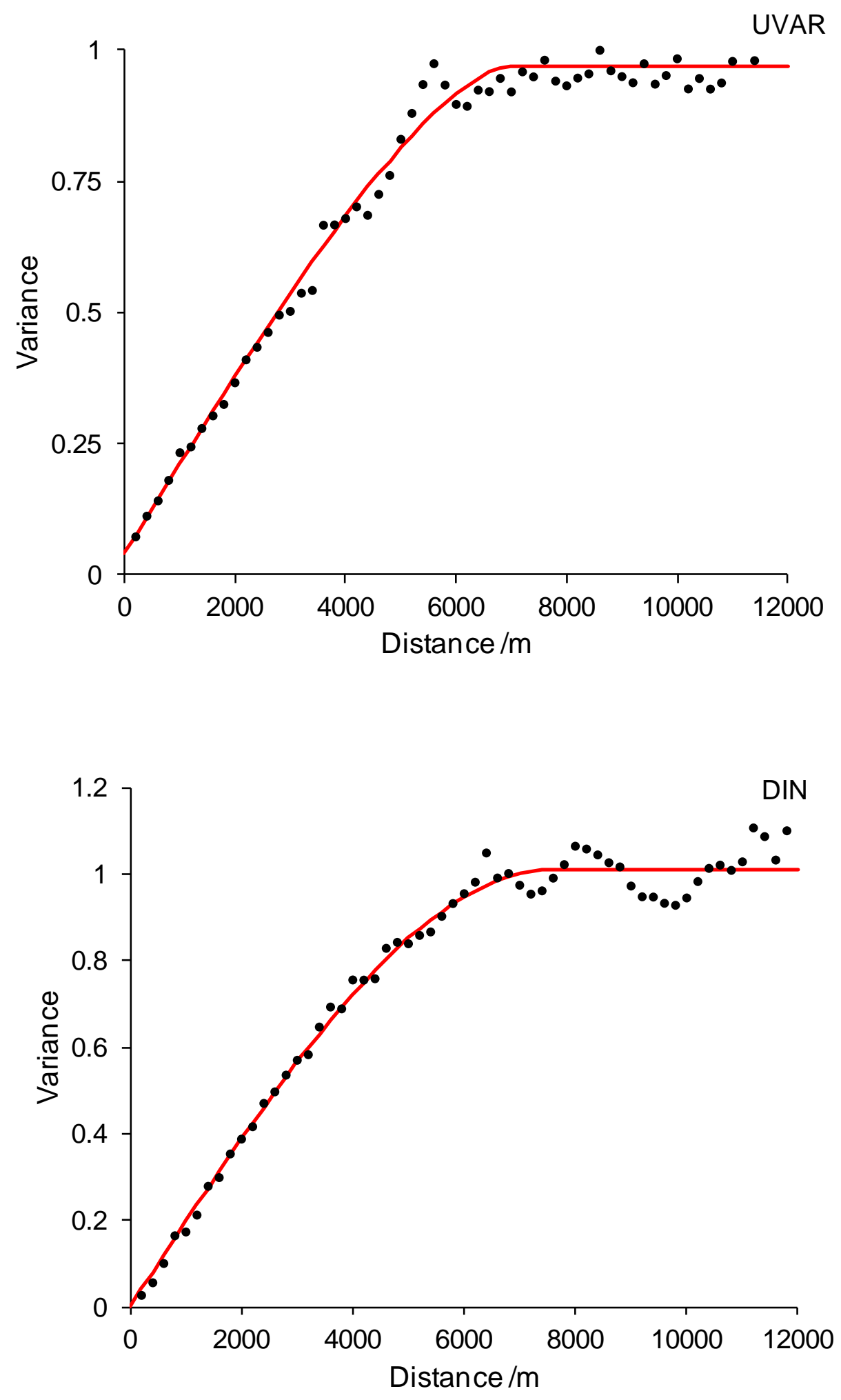


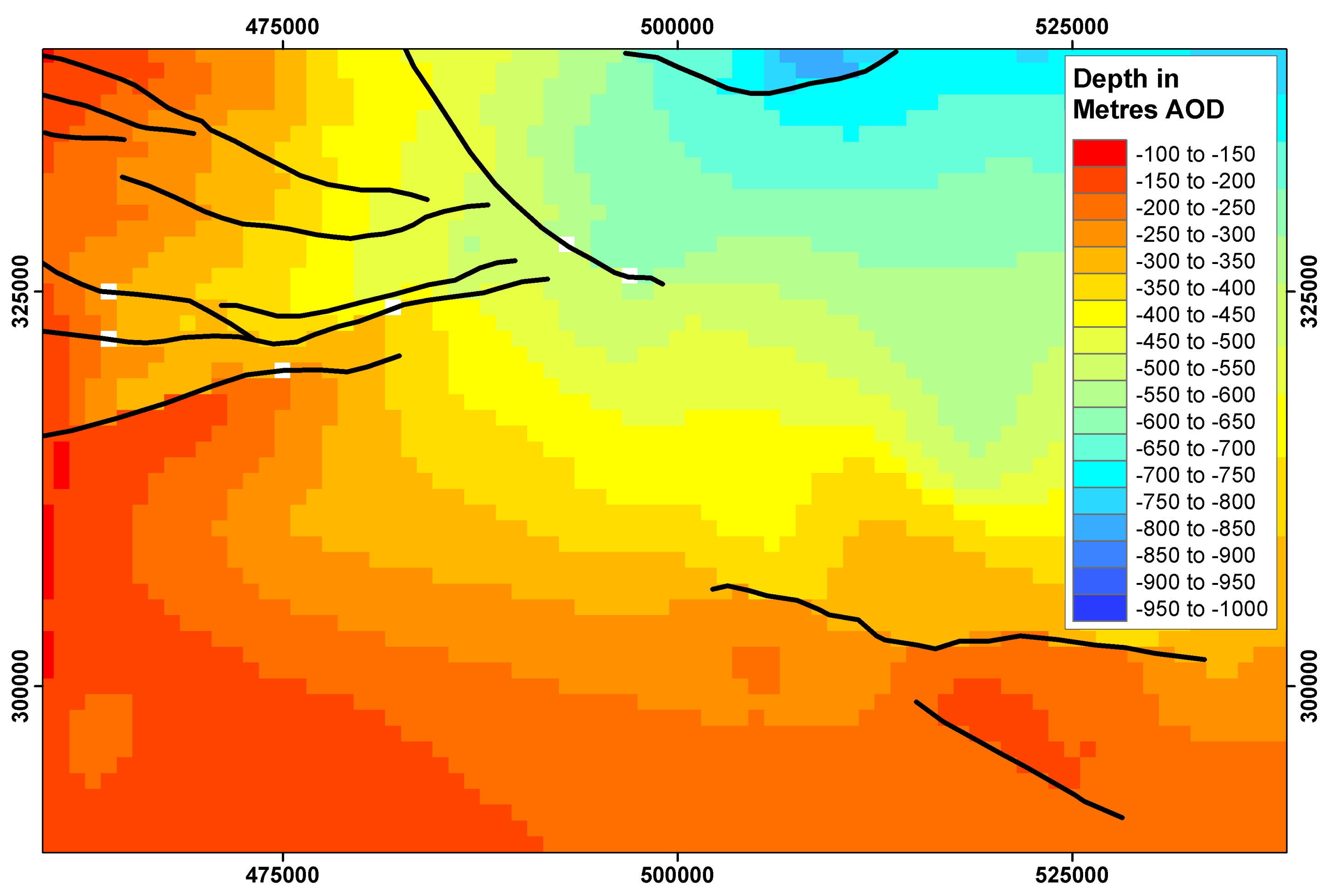




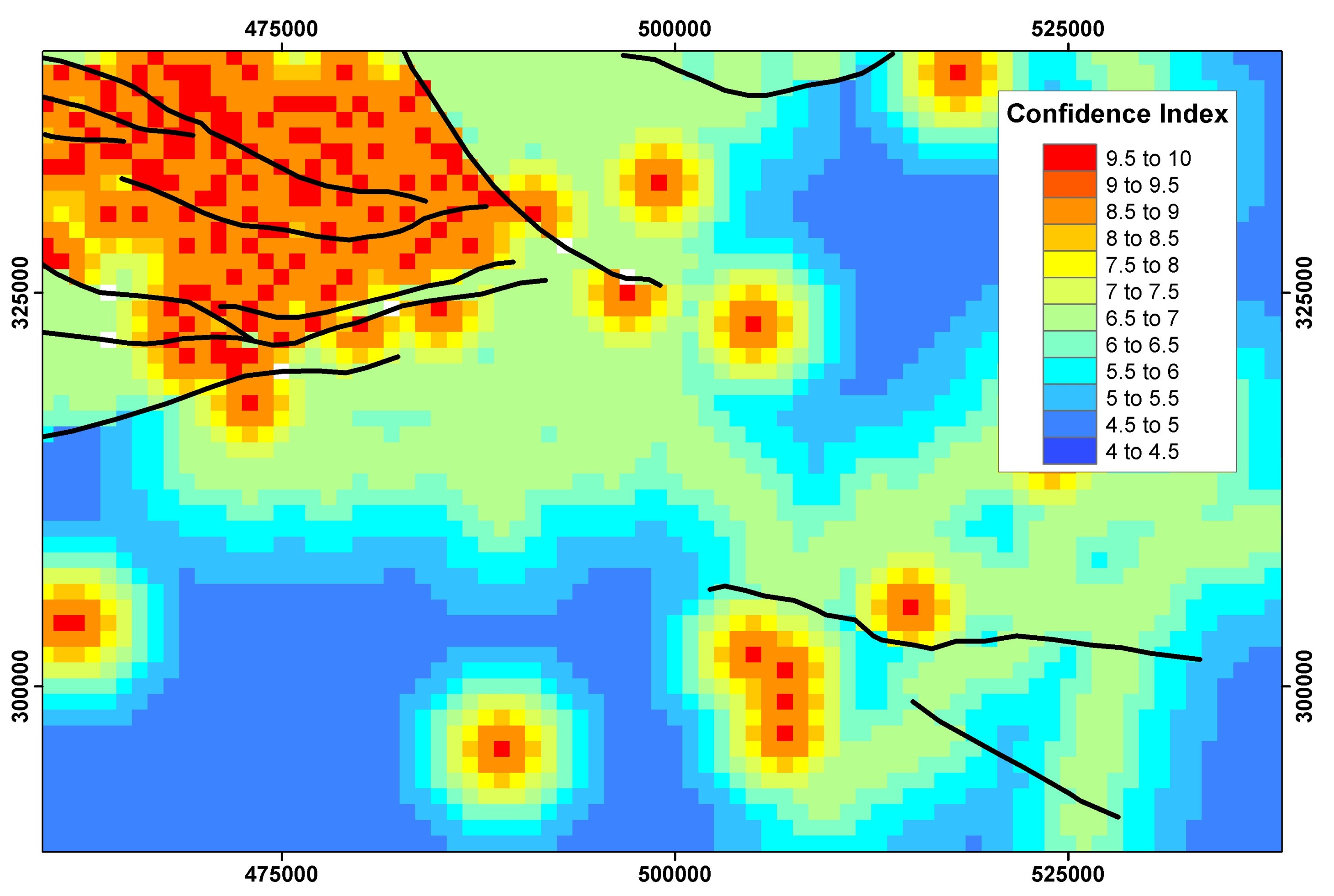




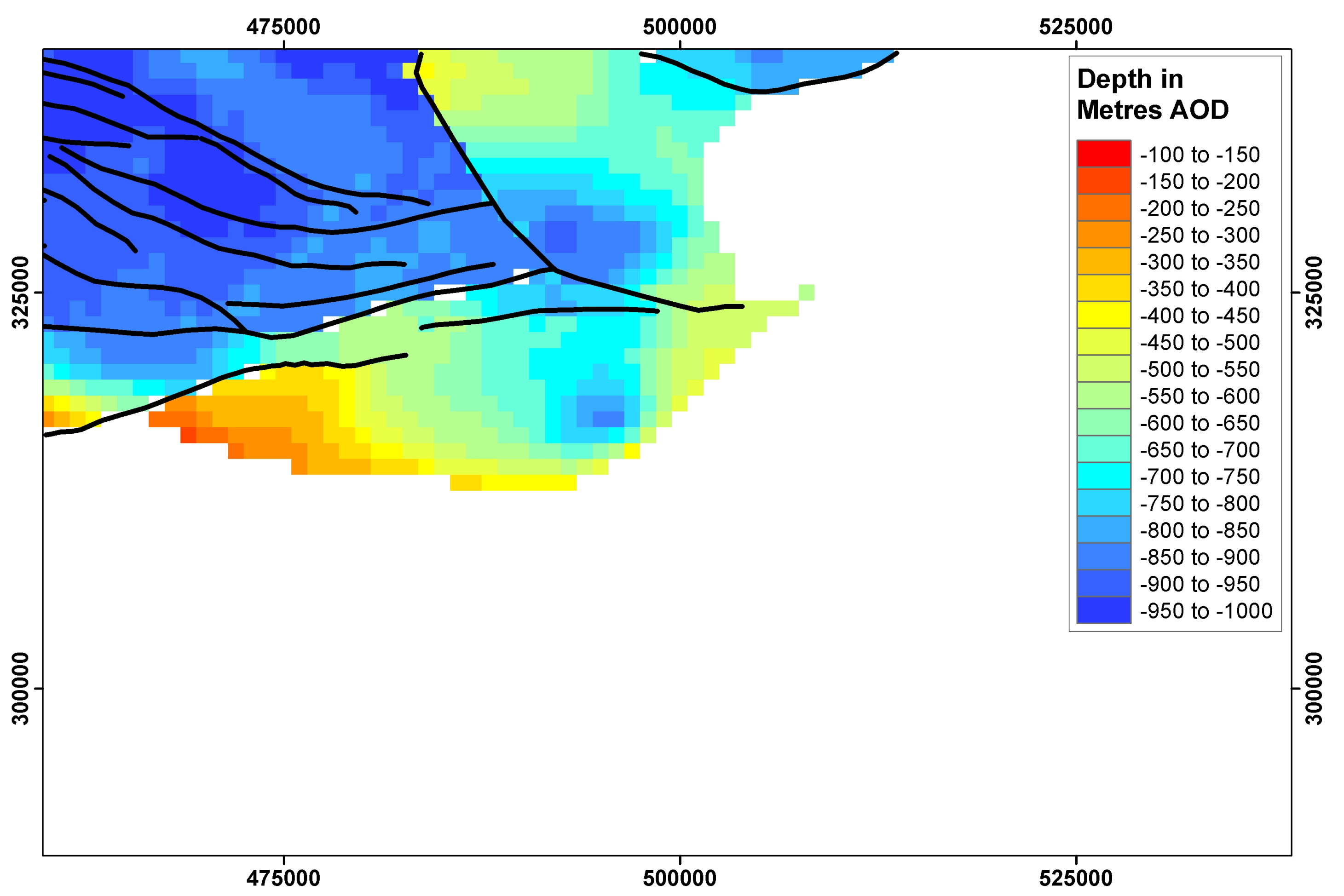




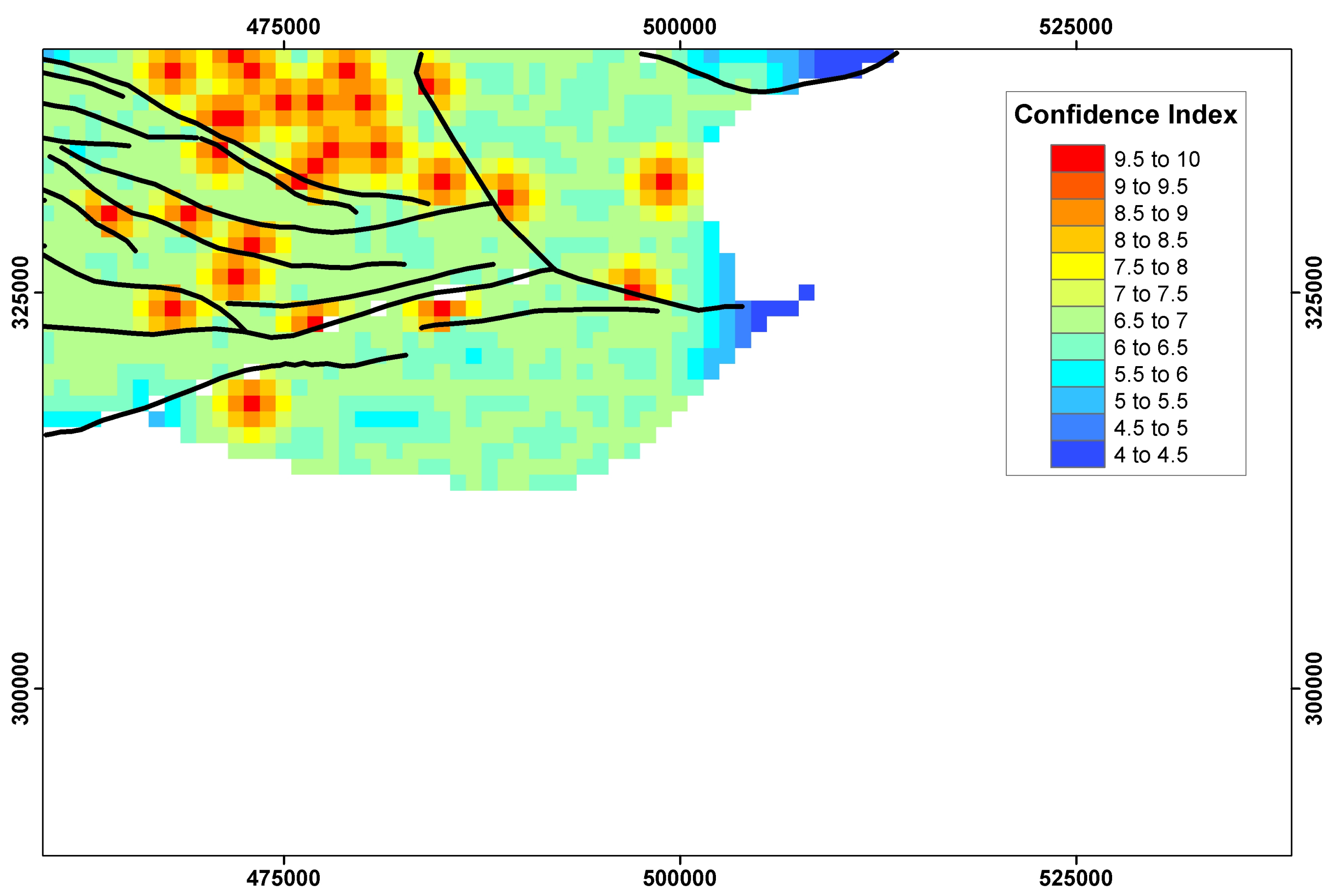


Table 1. Coefficient of determination for the trend surface for the geological surfaces of interest $\left(R^{2}\right)$, the inferred value of $c$ and parameters of the fitted variogram model.

\begin{tabular}{|l|l|l|l|l|l|l|}
\hline Surface & \multirow{2}{*}{$\begin{array}{l}\mathrm{R}^{2} \text { for fitted } \\
\text { trend } \\
\text { surface }\end{array}$} & $\begin{array}{l}\text { Inferred } \\
\text { strength of } \\
\text { trend, and } \\
\text { value of } c\end{array}$ & \multicolumn{4}{|l|}{\begin{tabular}{l}
\multicolumn{4}{l|}{ Parameters of fitted isotropic } \\
variogram model
\end{tabular}} \\
\cline { 3 - 7 } & & Model type & $g_{0}$ & $g_{1}$ & $\begin{array}{l}\text { range } \\
(\mathrm{m})\end{array}$ \\
\hline $\begin{array}{l}\text { Variscan } \\
\text { Unconformity } \\
\text { (UVAR) }\end{array}$ & 0.88 & Strong & Circular & 0.04 & 0.93 & 6899 \\
\hline $\begin{array}{l}\text { Top Dinantian } \\
\text { (DIN) }\end{array}$ & 0.66 & Moderate & Spherical & 0.0 & 1.01 & 7620 \\
\hline
\end{tabular}


Table 2. Elicited values of parameters for the confidence index in the study area.

\begin{tabular}{|c|c|c|c|}
\hline Parameter & Meaning of parameter & Elicited value & Comments \\
\hline$a$ & $\begin{array}{l}\text { Value of the confidence index at } \\
\text { the site of a borehole }\end{array}$ & 10 & $\begin{array}{l}\text { All boreholes regarded } \\
\text { as equal in quality }\end{array}$ \\
\hline$b$ & $\begin{array}{l}\text { Value of the confidence index at } \\
\text { the site of a seismic observation }\end{array}$ & 7 & $\begin{array}{l}\text { All seismic observations } \\
\text { regarded as equal in } \\
\text { quality }\end{array}$ \\
\hline$c_{1}$ & $\begin{array}{l}\text { Value of the confidence index at a } \\
\text { site remote from a borehole in the } \\
\text { absence of spatial trend. }\end{array}$ & 1 & \\
\hline$c_{2}$ & $\begin{array}{l}\text { Value of the confidence index at a } \\
\text { site remote from a borehole in the } \\
\text { presence of weak spatial trend. }\end{array}$ & 3 & \\
\hline$c_{3}$ & $\begin{array}{l}\text { Value of the confidence index at a } \\
\text { site remote from a borehole in the } \\
\text { presence of moderate spatial } \\
\text { trend. }\end{array}$ & 4 & \\
\hline$C_{4}$ & $\begin{array}{l}\text { Value of the confidence index at a } \\
\text { site remote from a borehole in the } \\
\text { presence of strong spatial trend. }\end{array}$ & 5 & \\
\hline
\end{tabular}

